\title{
Fabrication of Porous Structure of Electro-spun PVDF Fibres
}

\author{
Tao Zhang \\ The University of Manchester, United States \\ Email: zhangt2210@outlook.com
}

\begin{abstract}
Nano and porous materials with large surface area, high porosity, high surface reactivity, and strong adsorption properties have great application potential in energy and chemical industry, biological medicine, environmental science, defence engineering and numerous additional fields. Electrospinning is a convenient, direct and economical method of production of nano fibre materials. Porous fibre can be created by adjusting the process parameter of electrospinning or post processing after spinning. The introduction of porous structure into materials increases its surface area considerably and simultaneously enhances other properties. This indicates great potential for development in many fields and improves upon porous material application value. This project uses electrospinning to prepare polyvinylidene fluoride (PVDF) fibre and, post process, for PVDF porous fibre, utilising a combination of SEM, XRD and other tests to characterise properties. The effect and regularity of solution properties and processing parameters on prepared samples were studied along with experimental results and theoretical basis for preparation of electrospun PVDF fibres and fibres with porous structure. The main conclusions of this article are as follows:

1) Solvent concentration has a great influence on fibre morphology, enhancing PVDF concentration and resulting in increased regularity of fibre; meanwhile, fibre diameter will increase in correlation with the increase of concentration.

2) With the increase of acetone in DMF or NPM/acetone system, fibre morphology improves.

3) Assist solvent influences fibre morphology and samples created by DMF are superior to those created by NMP. There are fewer beads and fibres appear more regular.

4) Post processing parameter influences formation of pores, such as processing solvent, time, etc.
\end{abstract}

Keywords: Electrospinning, PVDF, porous structure, post processing, influence factor.

\section{Introduction}

\subsection{Research Background}

With the development of science and technology, human understanding of nature has gradually deepened and transferred from macro-world to micro-world. Developments in modern science and technology have resulted in higher requirements for performance of materials. The unique physical and chemical properties of nano materials, such as high specific surface area and small volume, lead to unique characteristics such as high catalytic activity and quantum size effect. This ensures wide use in the fields of catalysis, biomedicine, aviation, communication and automatic control. This has aroused the interest of researchers globally(Burda et al., 2005)(Chambers, 2010)(Whitesides, 2005)(Li and Xia, 2004). The concept of nanotechnology has become increasingly familiar to people since the late 1990s, and currently nano materials are increasingly used in people's daily work and life, effecting great change in everyday existence(Guo, Zhou and Lv, 2013). The $21^{\text {st }}$ century is evidently an era for production of new material due to rapid developments. Nano material has become a crucial feature to promote the development of contemporary science and technology. Scientists have successfully prepared a variety of different components of nanostructured materials. The structure of nano-materials has developed from zero-dimensional and one-dimensional, with many different internal forms and structures such as coreshell spheres/fibres, hollow spheres/fibres, multilayer spherical/fibres and multi-ball/fibres(Zhao and Jiang, 2009)(Sukitpaneenit and Chung, 2009)(Yee et al., 2010), to solid nanofibre and nanomicrospheres. These nanomaterials, with their special structure and unique properties, possess wide 
prospects for application, and have become increasingly common in recent years(Megelski et al., 2002).

\section{$1.2 \quad$ Aims of This Project}

Electrospinning technique is a spinning method in which polymer fibre or melt is subjected to spinning under static electricity to obtain continuous fibre. This method can create fibres from a few nanometres to tens of microns, and is a basic method for preparing nanofibre. Electrospun nanofibre membrane is widely used in the fields of filter material, biomedical functional materials, superhydrophobic and superabsorbent materials, sensors and polymer nanometre templates due to its high porosity, high specific surface area, high adsorption and filterability(Choi et al., 2003)(Choi et al., 2007)(Kim et al., 2004)(Gao et al., 2006).

Polyvinylidene fluoride (PVDF) has become a hotspot of gel polymer research as a result of its excellent electrochemical performance, strong and resilient structure, good bonding properties, high impact strength, tensile strength, creep resistance and good abrasion resistance, good chemical stability, high temperature resistance, oxidation resistance, chemical corrosion resistance, weatherability, resistance to radiation and other aspects relating to overall outstanding performance(Manuscript, no date)(Yee et al., 2007). With electrospinning technology for preparing porous polymer fibre possessing qualities such as high specific surface area, high adsorption performance, controllable fibre and pore size, PVDF can have wider applications for use in numerous fields(Greiner and Wendorff, 2007). This project focuses on influencing factors for PVDF films prepared by electrospinning and methods of adding porous qualities after post processing.

\section{Experiment}

Electrospinning is a novel method for preparation of polymeric ultrafine fibres. Compared with alternative methods, electrospinning is more convenient, simple, economical and effective, and is the only technique capable of continuous preparation of nano/micron fibre. To date, the electrospinning technique has been used for more than 100 spinning tests of polymer solution or melt, through choosing appropriate polymer materials, adjusting the appropriate parameters in electrospinning, postprocessing and modification of performance control and for the preparation of the fibre. The series of process parameters can affect characteristics of prepared electrospun fibres during the spinning process, as both beaded or uniform electrospun fibres or even droplets and particles may be prepared in the same spinning device by altering spinning parameters. Influencing factors include the following components: 1) polymer parameters, mainly polymer type and composition, solubility and molecular weight; 2) solvent parameters, mainly volatile solvent, boiling point, saturated vapour pressure and dipole moment of spinning solvent; 3) solution parameters, including solution concentration, viscosity, surface tension, electrical conductivity and solution temperature; 4) processing parameters, mainly including spinning voltage, receiving distance and spinning speed; 5) environmental parameters, referring to environment temperature, humidity and gas medium atmosphere.

To make fibre ultrafine is an important research priority in fibre science and engineering. Ultrafine polymer fibres prepared by electrospinning technology have a high specific surface area, high porosity, high surface activity, and strong adsorption and pore connection, thus electrospinning has great application value in many fields, and is being increasingly applied. In actual application, a critical factor is surface morphology of fibre. Fibre surface morphology and internal structure will alter as a result of adjusting process parameters of spinning either directly, or with post processing. Porous fibre has a higher surface area compared to typical electrospun fibres and their surface has higher activity and stronger adsorption, thereby improving performance in related fields; its scope of application will become broader and connected to processes such as ultrafiltration, catalyst carrier, biological tissue engineering and biological sensors etc. At present, relevant research indicates that induced phase separation is the preferred method of preparing porous fibres during the electrospinning process. There are 3 main considerations: 1) interactions between polymer and the solvent effect; 2) effects between polymer and non-solvent; 3) effects between polymer and blend components, interaction between copolymer composites. There is a considerable amount of existing research on the preparation of ultra-fine and porous fibre by electrospinning. However, preparation of PVDF porous fibres by electrospinning is at 
present not commonplace. The potential applications of porous PVDF fibre and the potential to broaden its range of application due to its excellent physical and chemical properties warrants further exploration.

\subsection{Preparation of Porous PVDF Fibres}

The main experimental process of preparing porous PVDF porous fibre is shown in Figure 3.1.

\section{Prepare different solvents}

\section{SEM photos to observe the}

morphology of the fibres

\section{Find out the best solvent and ratio, post process}

\section{SEM photos to find if there} are pores

\section{XRD FTIR DSC tests to figure out phases and molecules of the fibres}

Figure 3.1. Experiment plan.

\subsubsection{Apparatus and reagents}

The electrospinning device, supplied by Tongli Tech, Shenzhen, China, is mainly composed of three parts: the high-voltage power supply device, solution storage and injection device and a receiving device, wherein the high-voltage power supply can produce 0-30 KV. The injection pump can be installed on the screw injector composed of solution storage and injection device; the experimental device as shown below. There is also a heat device to adjust temperature and humidity during the process, it should be activated before spinning to ensure environment conditions remain constant in each group.

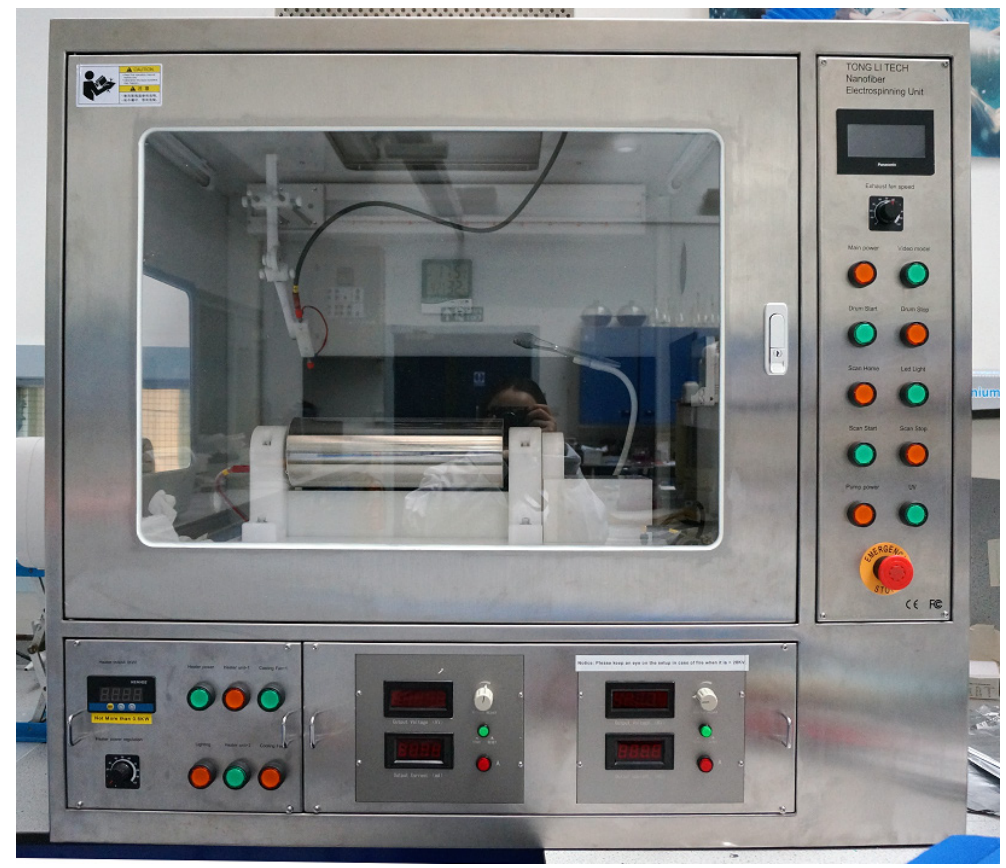

Figure 3.2. Electrostatic spinning device. 


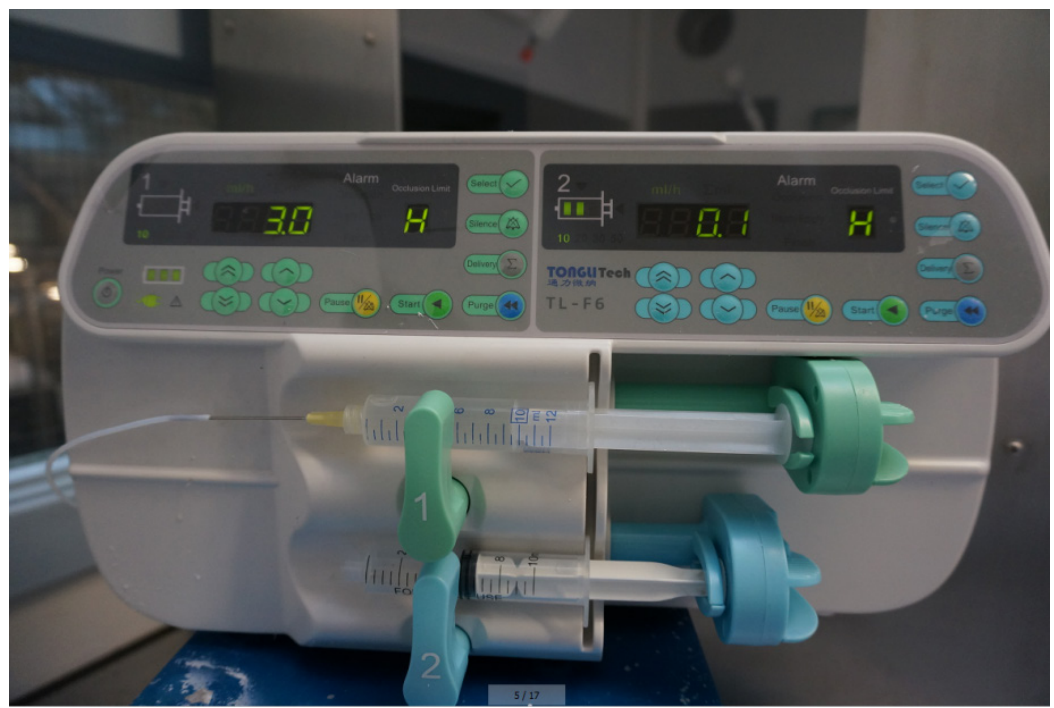

Figure 3.3. Injection device.

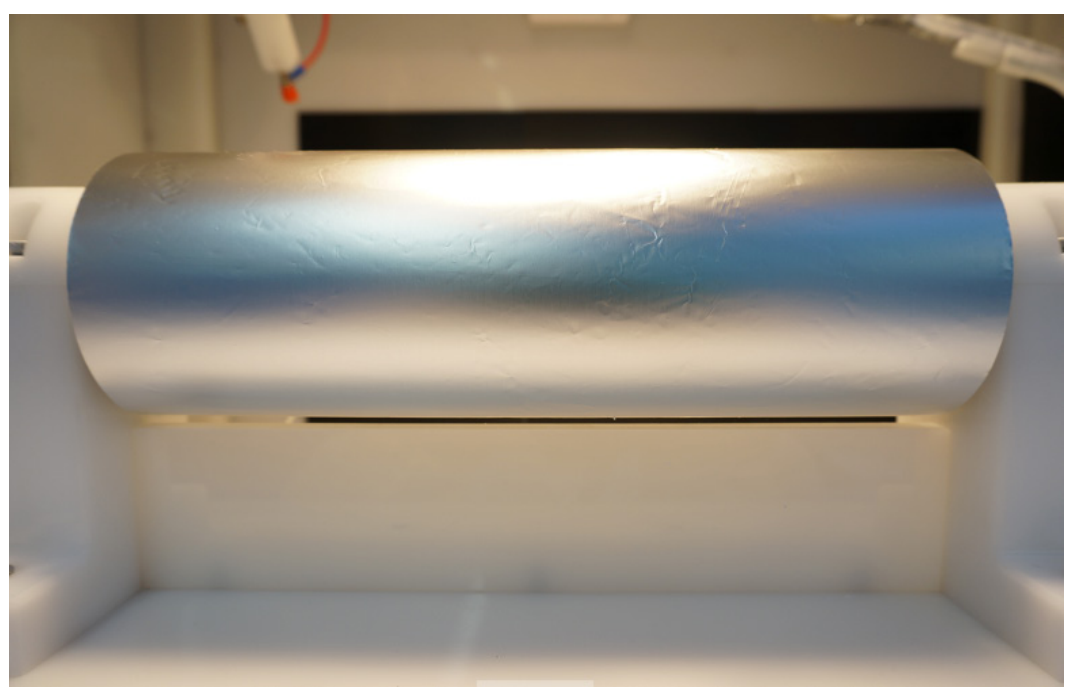

Figure 3.4. Receiving device.

\subsubsection{Experiment reagents}

In this article, Dimethylformamide (DMF), N-Methyl-2-pyrrolidone (NMP), Cellulose Acetate Butyrate $(\mathrm{CAB})$ and acetone are used as solvents to prepare PVDF mixture solutions for electrospinning. Main reagents are shown in Table 3.1.

Table 3.1. Main experimental reagents and materials.

\begin{tabular}{ccc}
\hline Reagents & Molecule weight & Purity \\
\hline PVDF & 534,000 & General purpose grade \\
NMP & 99.133 & General purpose grade \\
DMF & 73.09 & General purpose grade \\
Acetone & 58.05 & General purpose grade \\
CAB & 100,000 & General purpose grade \\
PVP & 40,000 & General purpose grade \\
\hline
\end{tabular}




\subsubsection{Preparation of spinning solution}

The preparation steps are as follows:

1) Use cylinder volume to obtain the specified amount of solvent according to experiment plan as illustrated above, decant into a $30 \mathrm{ml}$ solution bottle

2) Then use electronic analytical balance to weigh PVDF powder and place into the bottle with solvent in the last step

3) Add a magnetic rotor into the bottle, secure the bottle cap, then place a magnetic stirrer in the bottle to stir $24 \mathrm{~h}$ at $400 \mathrm{rpm}$ until PVDF powder is completely dissolved in the solvent

4) When solvent becomes clear and transparent, remove and wait for bubbles to disappear completely.

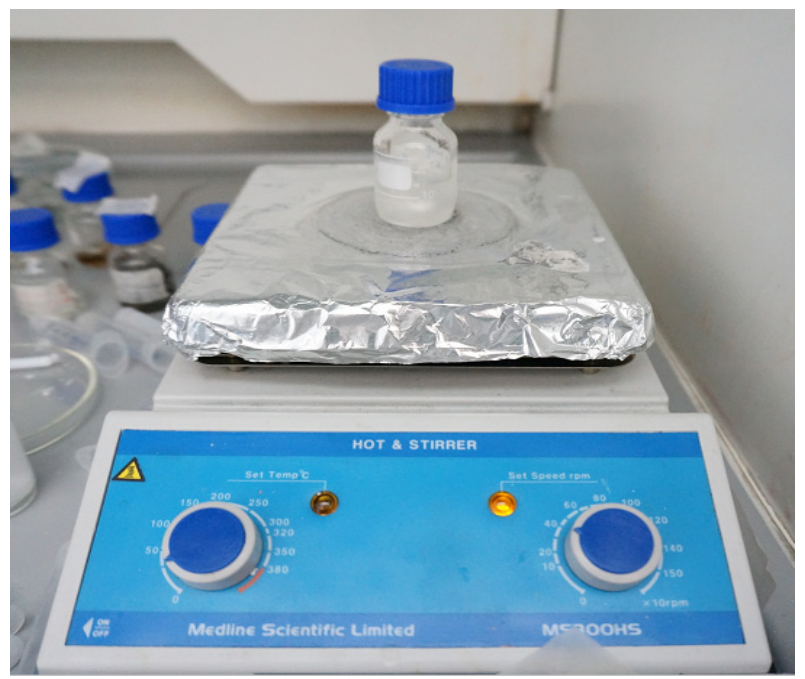

Figure 3.5. Solution bottle on stirrer.

\subsubsection{Electrospinning}

Firstly, the internal surface of the electrospinning device should be cleaned to ensure that the spinning process can be observed. A clean syringe is used to extract the specified amount of solution, then the syringe is set on the injection device, and injection needle is installed at the end of infusion tube and connect to spinning device. A $15^{*} 30 \mathrm{~cm}$ aluminium film is pasted on the receiving device, subsequently the spinning machine is activated, and injection speed is set. Next, adjust the distance between cleaning brushes and injection needle to ensure injection needle can be cleaned during the spinning process. After completing preparation process, adjust spinning parameters such as spinning voltage and width and commence spinning. Injection device will sound alarm when there is little solvent, at which point turn voltage off to 0 , open the door of the spinning machine and remove electrospun PVDF membrane for testing and analysing, then remove the syringe and infusion tube to clean remaining liquid for next use.

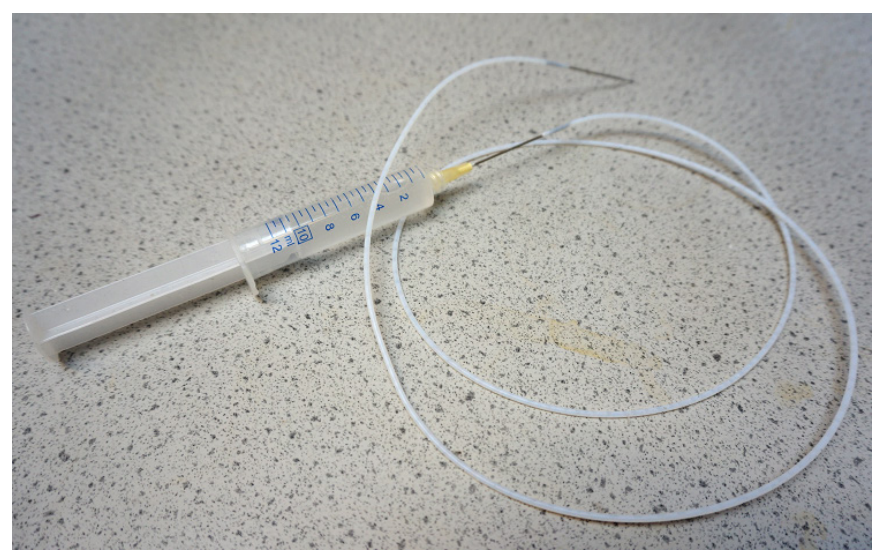

Figure 3.6. Clean syringe with infusion tube. 


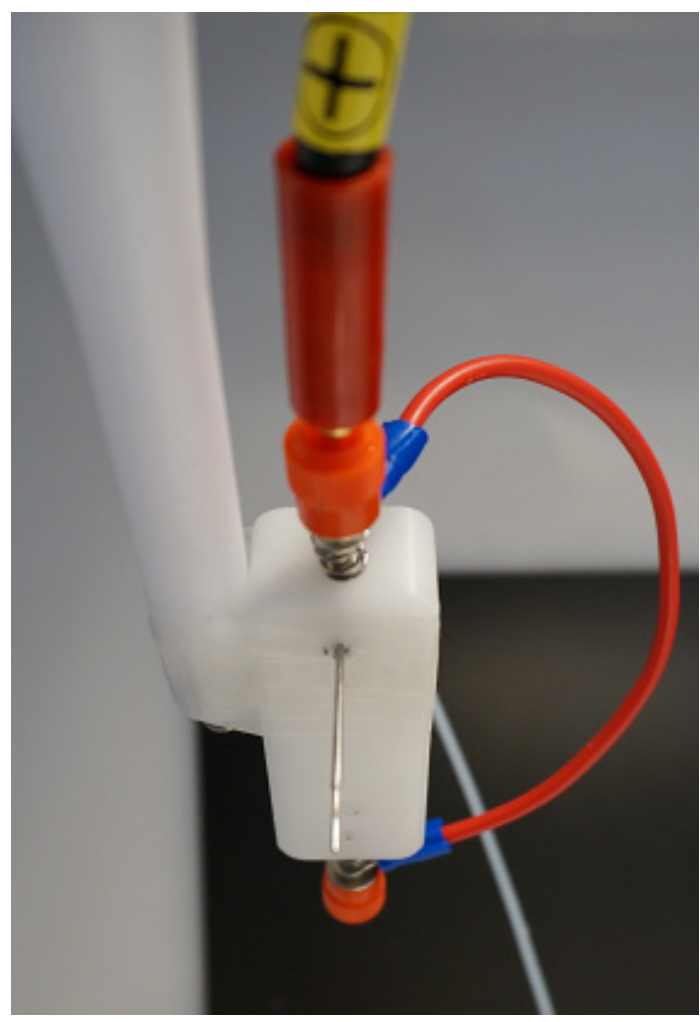

Figure 3.7. Injection needle.

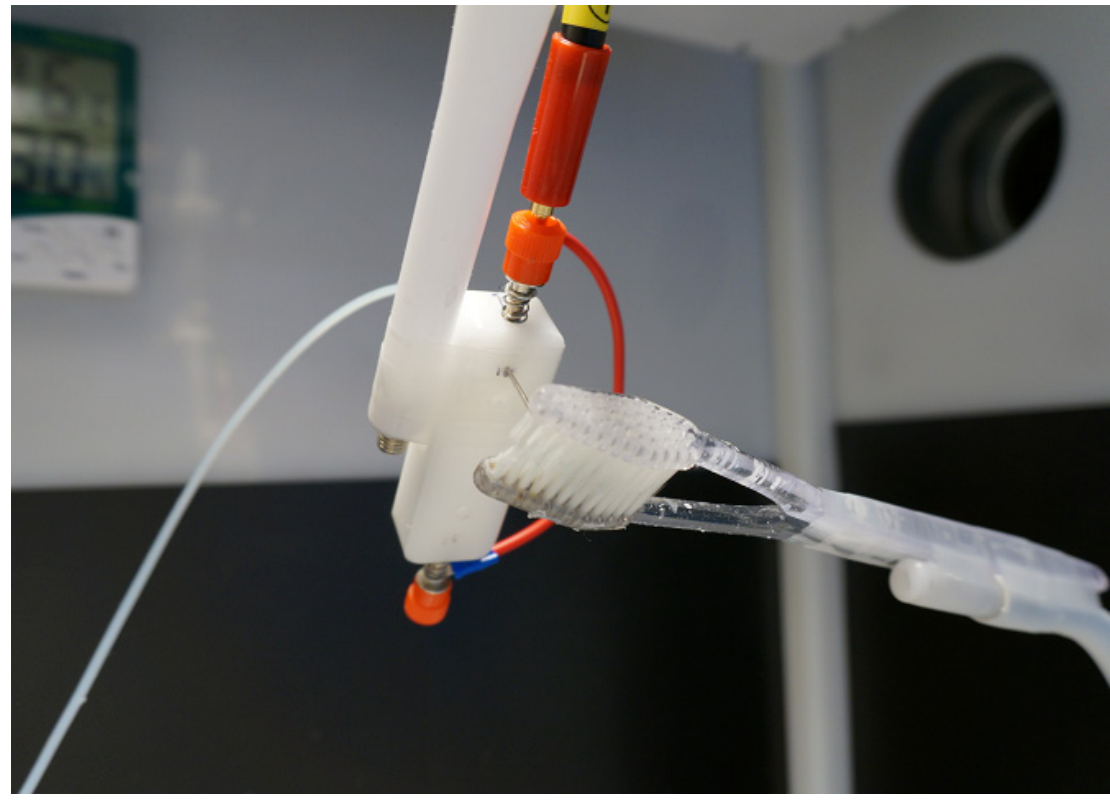

Figure 3.8. Injection needle must be able to go through cleaning brushes.

\subsubsection{Preparation of blended PVDF nanofibre}

Phase separation is used on electrospun polymer blend fibre to prepare porous PVDF fibres. Cellulose Acetate Butyrate (CAB) or Polyvinyl pyrrolidone (PVP) is added to PVDF solution and prepared into electrospun polymer composite fibre. Induced phase separation occurs between pore forming agent and PVDF during electrospinning process, then post process composite fibre is obtained by removing added porogen from the composite fibre, and porous PVDF fibre with a large number of hierarchical pore structure is prepared. Through characterisation of the prepared PVDF porous fibre preparation (surface 
morphology, pore size and distribution, etc.), the influence of type and amount of pore forming agent on preparing porous PVDF fibres can be studied, which has significance for the fabrication of electrospun porous PVDF superfine fibre and its application.

The first stage is to prepare certain proportion of DMF and acetone as solvent and PVDF as solute, then add CAB or PVP in PVDF solution as porogen. The spinning process is the same as stated in chapter 3.1.4 of this paper. Treat the prepared with different methods of obtaining porous structure. Finally, field emission scanning electron microscopy (FESEM), and XRD are used to characterise the morphology and other properties of PVDF porous fibre.

\subsubsection{Post process}

Cut small samples of prepared films and place into a beaker filled with ethanol immersion: Deionized water $=1: 1$ (volume ratio) for 10 minutes then stand for different times, wash 3 times with deionised water, remove the residual product in the samples then remove samples and place in a clean Petri dish in room conditions until the quality of samples are no longer shifting. The dried fibres are retained for subsequent testing and characterisation.

\subsection{Characterisation of PVDF Fibres}

\subsubsection{SEM tests}

Cut a small piece from prepared sample and place it on SEM sample stub, then use small surface spraying ion sputter to coat. Put the processed sample stub into vacuum environment. Observe fibre morphology by scanning electron microscopy (SEM) with different magnifications, high magnification for observing overall morphology of PVDF film and low magnification for single fibre observation, resulting in simultaneous image acquisition.

\subsubsection{XRD tests}

$\mathrm{X}$-ray diffraction (XRD) is a method of obtaining polymer morphology information and can be used for material identification, structure identification, description and characterisation of crystalline state, crystal structure parameters of orientation, molecular conformation and molecular motion information determination. By using X-ray effect in crystalline and amorphous material, the characteristics of a sample crystal structure can be recorded through diffraction angle and intensity, and can acquire a wide range of information concerning solid polymer crystallisation. Sample size should be $2.5^{*} 2.5 \mathrm{~cm}$.

\subsubsection{DSC tests}

Differential scanning calorimetry (DSC) is one of the most widely used thermal analysis techniques. It is related to the mechanical and thermal properties of the sample, including glass transition temperature $(\mathrm{Tg})$, melting temperature (Tm), crystallisation temperature $(\mathrm{Tc})$, specific heat $(\mathrm{Cp})$ and enthalpy. The shape of the molten peaks on the DSC curve can reveal data about grain size distribution, and the enthalpy of melting can give intelligence regarding crystallinity. The influence of impurities and humidity can also be obtained by using DSC. The crystallisation temperature, crystallisation rate and influence of nucleating agent and recycled material can be obtained in program controlled cooling. The second heating curve can influence the material processing technology and preparation condition.

In this test, ramp rate is $10^{\circ} \mathrm{C} / \mathrm{min}$ from room temperature to $220{ }^{\circ} \mathrm{C}$ and is applied for 3 minutes, then decreases by $10{ }^{\circ} \mathrm{C} / \mathrm{min}$ to $40{ }^{\circ} \mathrm{C}$, then $10{ }^{\circ} \mathrm{C} / \mathrm{min}$ to $220^{\circ} \mathrm{C}$. Sample weight should be $3-5 \mathrm{mg}$.

\section{$3 \quad$ Results \& Discussion}

\subsection{SEM Test}

\subsubsection{Effects of PVDF concentration}

In order to study the influence of spinning parameters on electrospun fibre, single variables such as voltage, solution concentration and solvent ratio are changed while other conditions remain unchanged, spinning rate is $3 \mathrm{ml} / \mathrm{h}$, voltage is $18 \mathrm{KV}$, temperature is $30^{\circ} \mathrm{C}$ and humidity is $25 \%$. Specific experimental numbers and parameters are shown in Table 4.1. 
Table 4.1. Pure PVDF electrospinning parameters.

\begin{tabular}{ccccc}
\hline Number & PVDF/g & DMF/g & NMP/g & Acetone $/ g$ \\
\hline A1 & 0.5 & 3.0 & & 7.0 \\
A2 & 1.0 & 3.0 & & 7.0 \\
A3 & 0.5 & & 3.0 & 7.0 \\
A4 & 1.0 & & 3.0 & 7.0 \\
\hline
\end{tabular}

The concentration of electrospinning solution is a significant parameter that affects morphology and diameter of fibres prepared by electrospinning. Typically, as the concentration of polymer solution increases, the diameter of fibres increases accordingly. SEM tests of group A are shown as follows, the magnification is $1000 \mathrm{X}$.
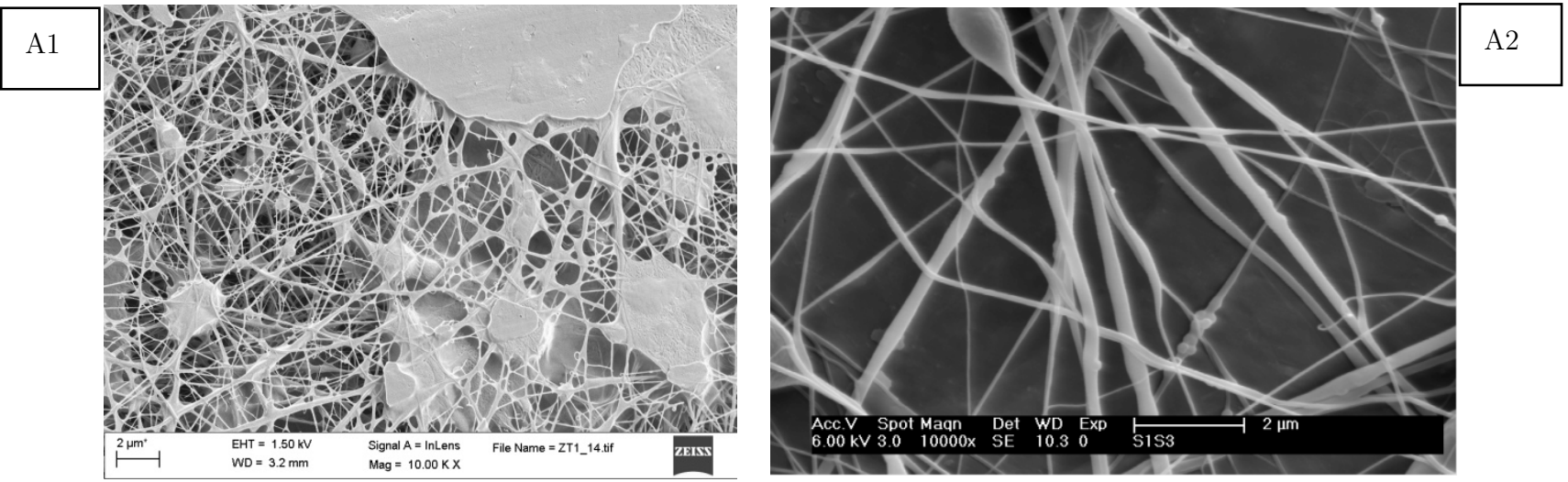

Figure 4.1. SEM images of pure PVDF fibres prepared with DMF. (A1: 0.5 g PVDF 3.0 g DMF 7.0 g Acetone. A2: 1.0 g PVDF 3.0 g DMF 7.0 g Acetone)
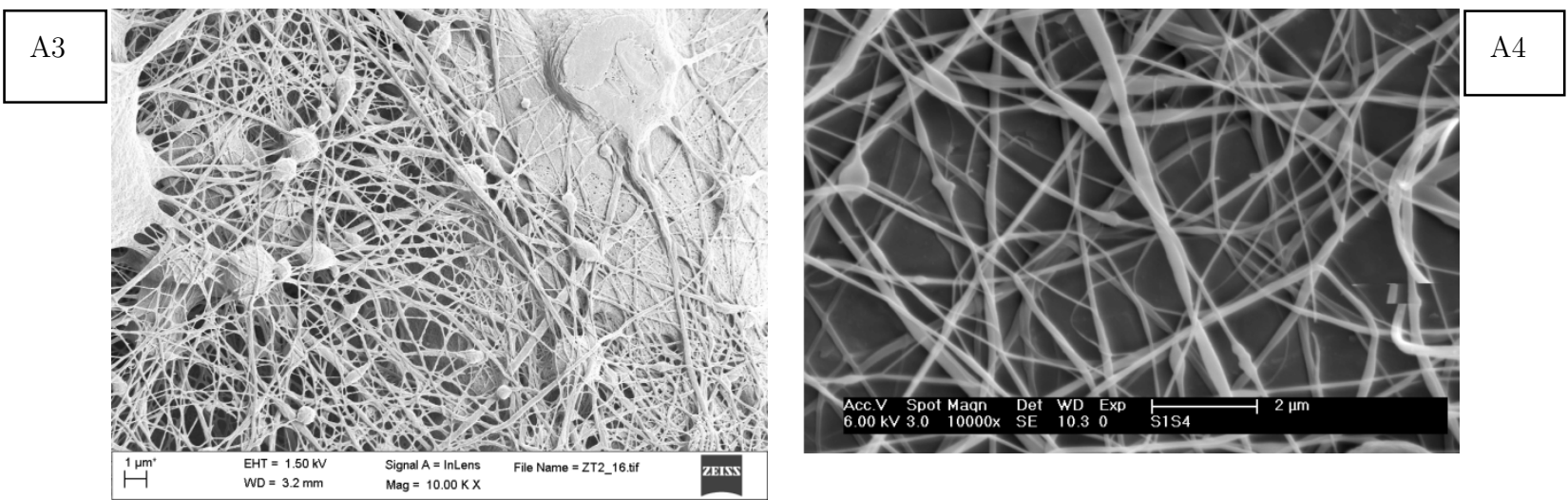

Figure 4.2. SEM images of pure PVDF fibres prepared with NMP.(A3: 0.5 g PVDF 3.0 g NMP 7.0 g Acetone A4: 1.0 g PVDF 3.0 g NMP 7.0 g Acetone)

It can be seen from A1 and A3 that when mass concentration of PVDF solution is less than 5\%, the prepared fibres only obtain a small number of polymer beads because concentration and viscosity of the electrospinning solution are relatively low. Polymer solution formed during the spinning process is subjected to electric field force stretching in the electrostatic field due to the fact that there is either no entanglement or that entanglement between molecular chains is not sufficient to effectively resist electric field force. Simultaneously, the polymer molecular chain tends to shrink due to the viscoelastic effect, resulting in agglomeration of molecular chains of the polymer and the final formation of aggregated beads. As shown in A2 and A4, when mass concentration of PVDF solution is approximately 10\%, both fibres and beads exist in electrospun polymer film as the spinning solution concentration and viscosity have exceeded contact concentration, the degree of entanglement between molecular chains increases, 
and relaxation time of polymer solution jet in the electrospinning process is increased by the stretching action of electric field force. The entangled molecular chains can be moved along the axial direction of jet which effectively resists the effects of electric field force, so the molecular chain in the jet stream cannot be broken easily, and fibre structure is continuous. Additionally, due to the fact that the jet flow by electric field force during the process is unbalanced in terms of direction, molecular chain of coorientation is not consistent, so some beads remain, and polymer fibre and beads coexist in prepared film. In essence, the appearance of bead structure is due to the Rayleigh instability caused by viscoelasticity of polymer. When the concentration of polymer reaches a certain level, it is possible to obtain fibre structure where surface is smooth and has a relatively uniform diameter distribution, because the concentration and viscosity of the solution are sufficiently high and molecular chains in the solution are greatly entangled during the electrospinning process. The action of jetting force is relatively uniform, and the co-orientation of the molecular chain is consistent. Therefore, bead structure disappears, and all polymer fibres are obtained after solvent is volatilised.

\subsubsection{Effects of solvent composition}

Polymer chains in solution are separated from each other by the action of their solvent. During the electrospinning process, polymer solution forms a charged jet stream which is highly stretched by electric field force. Polymer chains are rearranged and oriented in this process while solvent in polymer jets is gradually volatilised and jets are gradually solidified to deposit onto the receiving device in fibre structure. In this process, properties of the solvent used, such as volatility, electrical conductivity, dielectric constant, and solubility, will have an effect on the process of electrospinning, which in turn affects the shape of polymer fibre morphology and diameter. The solubility of solvent to polymer is related to the entanglement of polymer molecular chain in solvent. For a specific polymer, its solvent can be divided into effective solvent and non-solvent; the tension and viscosity of same mass fraction polymer in good solvent and non-solvent are also different, so the morphology and diameter distribution of prepared fibres can be varied by adjusting the ratio of the blended solvent in electrospinning solution. In this group, spinning rate is $3 \mathrm{ml} / \mathrm{h}$, voltage is $18 \mathrm{KV}$, temperature is $30{ }^{\circ} \mathrm{C}$ and humidity is $25 \%$. Spinning parameters are shown in Table 4.2 .

Table 4.2. Experimental parameters and numbers.

\begin{tabular}{ccccc}
\hline Number & PVDF $/ g$ & DMF $/ g$ & NMP $/ g$ & Acetone $/ g$ \\
\hline B1 & 1.0 & 2.0 & & 8.0 \\
B2 & 1.0 & & 2.0 & 8.0 \\
\hline
\end{tabular}

PVDF solutions with different solvent ratio are electrospun and PVDF fibres are collected. The surface was sprayed and observed by SEM. The SEM images were shown in Figure 4.3, the magnification is $1000 \mathrm{X}$.
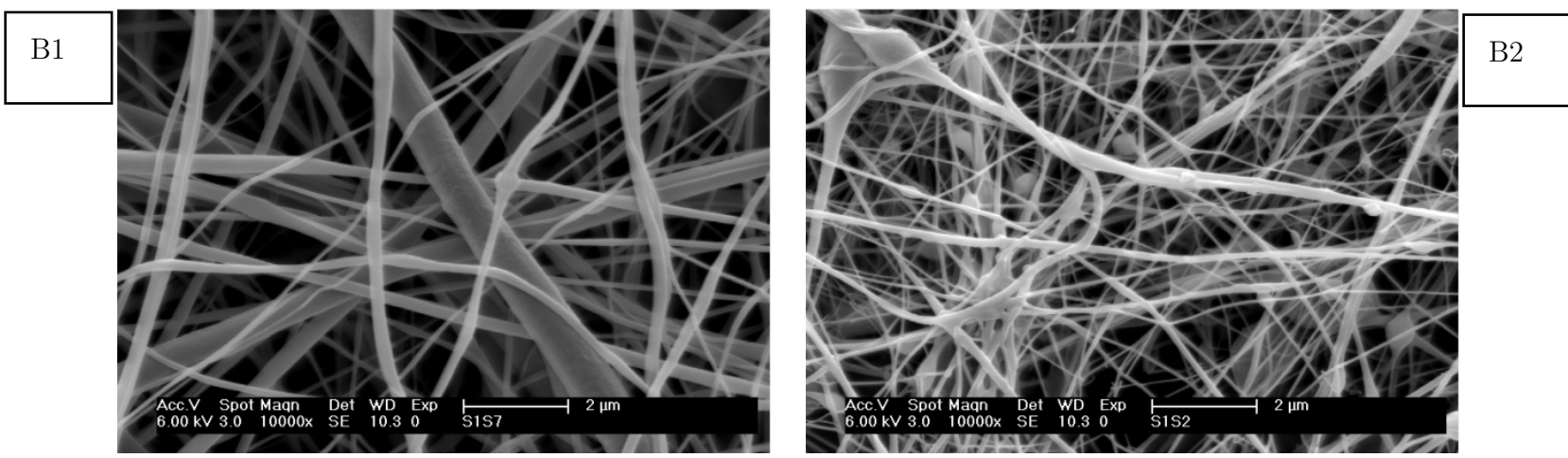

Figure 4.3. SEM images of same amount of PVDF fibres prepared with different solvent.(B1: $1.0 \mathrm{~g}$ PVDF $2.0 \mathrm{~g}$ DMF 8.0 g Acetone B2: 1.0g PVDF $2.0 \mathrm{~g}$ NMP $8.0 \mathrm{~g}$ Acetone)

By comparing A2, B1 and A3, B2, it can be concluded that when the ratio of NMP and DMF is high, fibre with beads is prepared. When the content of acetone in solvent increases, fibre becomes more 
uniform, as acetone has low boiling point $\left(65^{\circ} \mathrm{C}\right.$ ) and strong volatility (saturated vapor pressure 30.8 $\mathrm{kPa}\left(25^{\circ} \mathrm{C}\right)$, so by increasing the content of acetone in mixed solvent, the volatility of the polymer increases. During the electrospinning process, the majority of solvent contained in polymer jet stream is volatilised and only a small amount of the remaining solvent can reach the receiving device. This inhibits molecular chain viscoelastic contracting and shrinking to form bead structure, and only a small number of beads appear at interweave points.

By comparing plates A1, A2 in group A and B1 in group B, it can be deduced that when DMF is used as solvent, there are fewer beads in fibres and the morphology is more uniform, and fibre diameter is apparently larger compared to when NMP is used as a solvent. This indicates that although both DMF and NMP are effective solvents for PVDF, it is more productive to use DMF as a solvent in electrospinning.

\subsubsection{Effects of different porogens}

Observing the fibres prepared by electrospinning with different porogens to discover which porogen is more suitable for post processing can be achieved through studying fibre diameter and surface distribution. Spinning rate is $3 \mathrm{ml} / \mathrm{h}$, voltage is $18 \mathrm{KV}$, temperature is $30{ }^{\circ} \mathrm{C}$ and humidity is $25 \%$. Specific experimental numbers and parameters are shown in Table 4.3.

Table 4.3. Experimental parameters and numbers.

\begin{tabular}{cccccc}
\hline Number & PVDF $/ \mathrm{g}$ & $\mathrm{CAB} / \mathrm{g}$ & $\mathrm{PVP} / \mathrm{g}$ & $\mathrm{DMF} / \mathrm{g}$ & Acetone $/ \mathrm{g}$ \\
\hline $\mathrm{C} 1$ & 0.5 & 0.5 & & 2.0 & 8.0 \\
$\mathrm{C} 2$ & 0.5 & & 0.5 & 2.0 & 8.0 \\
\hline
\end{tabular}

SEM images are shown as follows, the magnification is 10000X.
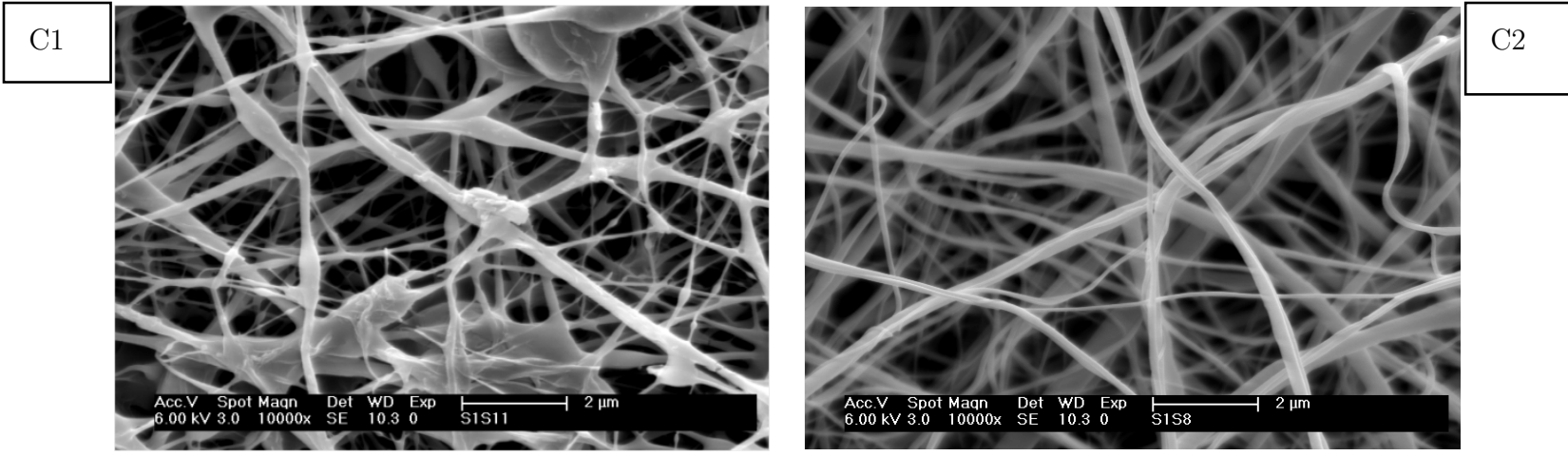

Figure 4.4. SEM images of PVDF nanofibres with porogen.(C1: 0.5 g PVDF 0.5 g CAB 2.0 g DMF 8.0 g Acetone C2: 0.5 g PVDF 0.5 g PVP 2.0 g DMF 8.0 g Acetone)

By comparing $\mathrm{C} 1$ and $\mathrm{C} 2$, it is evident that the fibre prepared with PVP has superior morphology to that prepared with $\mathrm{CAB}$. The diameter of fibre and web morphology are more regular, fibres orient in the same direction and fewer beads appear. Thereby, PVP is chosen as the porogen for subsequent experiments.

\subsubsection{Effects of porogen with different concentration}

After identifying which reagent can be used as porogen, effects of porogen concentration on fibre morphology should be understood; experiment parameters and numbers are listed in Table 4.4.

Table 4.4. Experimental parameters and numbers.

\begin{tabular}{ccccc}
\hline Number & PVDF $/ g$ & PVP $/ g$ & DMF $/ g$ & Acetone/g \\
\hline D1 & 0.25 & 0.5 & 2.0 & 8.0 \\
D2 & 0.5 & 0.25 & 2.0 & 8.0 \\
\hline
\end{tabular}


SEM images are shown as follows, the magnification is 10000X.
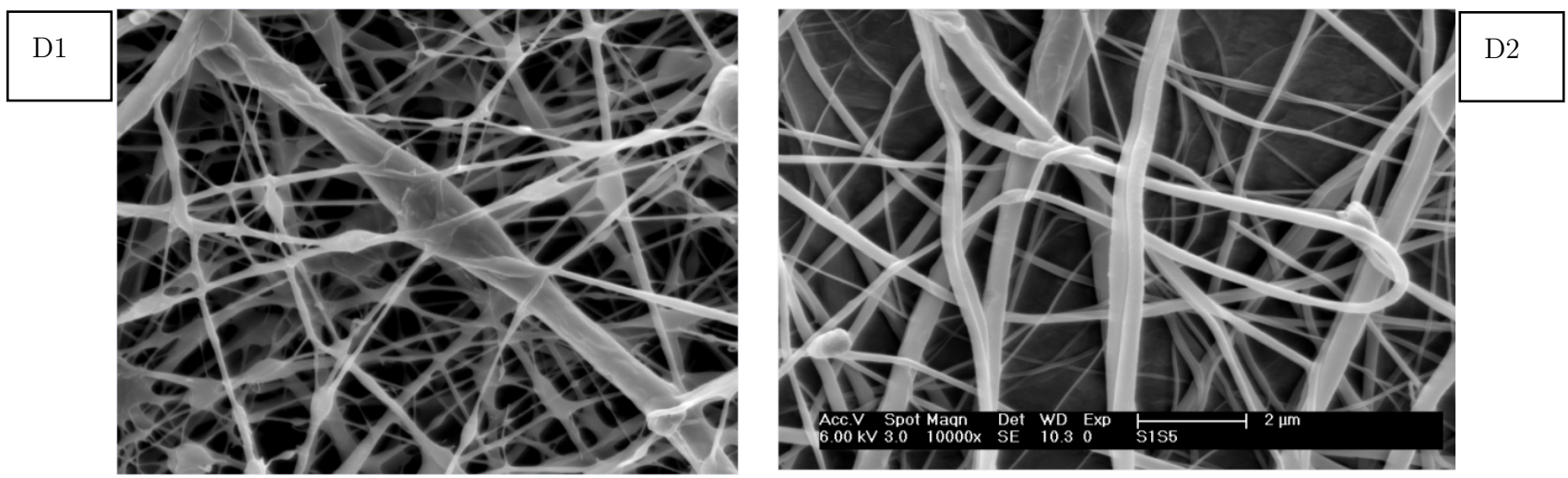

Figure 4.5. SEM images PVDF/PVP blended fibres with different blend ratio (magnification 10000X). (D1: 0.25 g PVDF 0.5 g PVP 2.0 g DMF 8.0 g Acetone. D2: 0.5 g PVDF 0.25 g PVP 2.0 g DMF 8.0 g Acetone)

By comparing D1, D2 with C2 in the previous section, it is apparent that when PVDF concentration is lower than PVP, fibre diameter is not even, most fibres are ultra-fine though a small number may be thick, and the majority of fibres show presence of beads. However, when PVDF concentration is higher than PVP, fibre diameter becomes notably uniform. In summary, when the ratio of PVDF:PVP is greater than 1, fibre morphology becomes ideal and suitable for post processing.

\subsubsection{Post processing}

Undertake SEM tests on post processed samples, with the variable in post processing that of time. Sample E1 is processed for 1 day, sample E2 is processed for 3 days and sample E3 is processed for 7 days. SEM photos are shown as follows, the magnification is $10000 \mathrm{X}$ in E1 and E3, and 200.00K in E2.

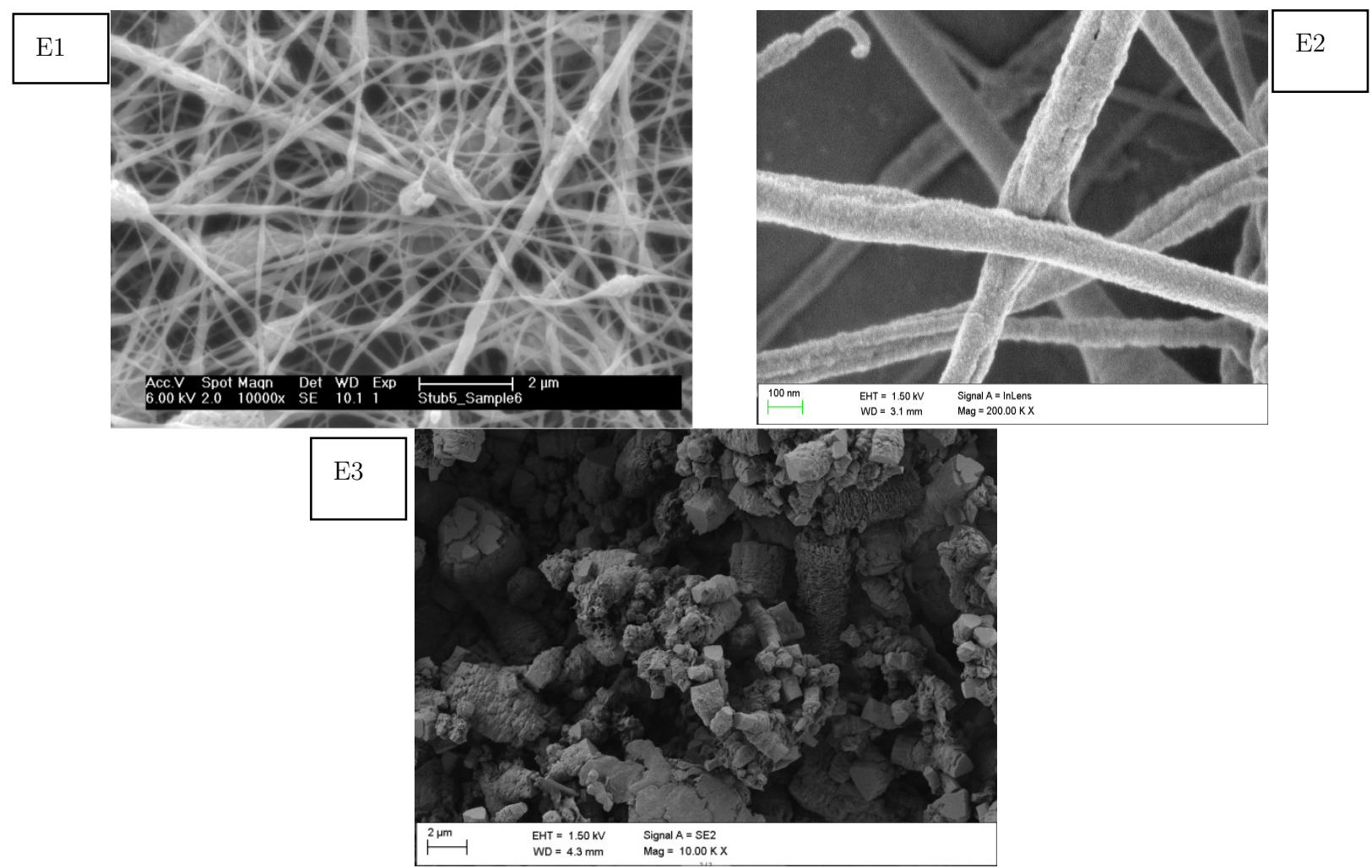

Figure 4.6. SEM images of PVDF/PVP fibres processed for different days.(E1: processed for 1 day E2: processed for 3 days E3: processed for 1 week) 
By observing SEM images in Figure 4.6, pores can be seen in beads in image E1, higher magnification of pores in beads is shown in Figure 4.7, the magnification is 50000X.

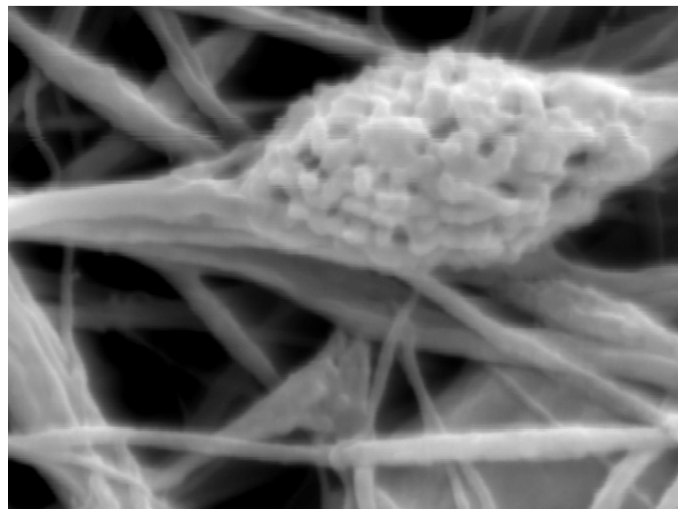

Figure 4.7. SEM images of pores in PVDF/PVP fibres processed for 1 day.(magnification 50000X)

According to the images above, when fibres are processed for 1 day, there is no pore structure in fibre, but the reason for porous structure in beads may be due to the fact that during the spinning process, the beads have less tensile force caused by electric force, so the crystallisation degree is not as high. Phase separation will occur more readily in this process, which will lead to recrystallisation and result in a porous structure. For fibres processed for 7 days, the fibres cannot retain their original morphology and become cubic, this may occur because processing time is too long and fibres may react with ethanol or become oxidised after being soaked in water.

\section{$3.2 \quad$ XRD Test}

In order to study the crystallisation of processed PVDF fibre, XRD is used as the test method. PVDF has multiple crystals in which $\beta$-phase is mainly porous structure, and the diffraction peaks appear largely between $2 \theta \approx 20.3$ degrees. This test is used in E2 and shown as follows.

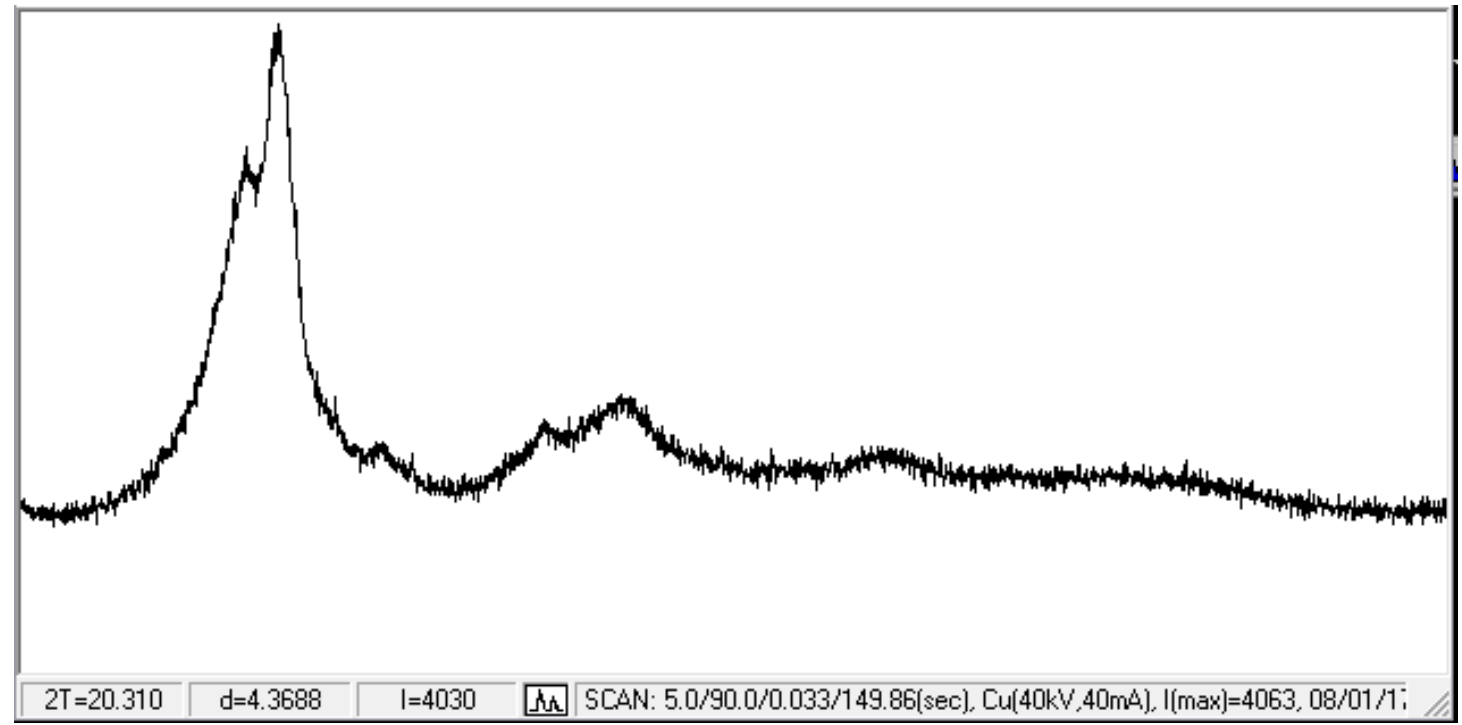

Figure 4.8. XRD test on E2.

It can be seen from Figure 4.8 that the highest peak in XRD test is $2 \theta=20.310$, indicating that the post processed fibre does have phase induction. 


\section{Conclusion \& Future Work}

\subsection{Conclusion}

This project prepared PVDF fibre and PVDF porous fibre by electrospinning, using a combination of FESEM, XRD and other test methods to characterise the influence of solution properties and processing parameters on prepared samples. Providing a theoretical and experimental basis for preparation of PVDF fibre and PVDF porous fibre, the main conclusions are as follows:

1) Electrospun PVDF fibre: with the increase of the solution concentration, the prepared fibre diameter increases, and fibre surface morphology is enhanced. When solution concentration is low, bead structure appears and subsequently gradually disappears with the increase of solution concentration; with the increase of acetone in DMF/acetone mixed solvent, fibre diameter increases.

2) Electrospun PVDF blend fibres: when spinning PVDF/CAB and PVDF/PVP blend polymer solution, $\mathrm{PVP}$ is more suitable than $\mathrm{CAB}$ as a bicomponent. By using ethanol/water mixed solvent to remove PVP, phase induction takes place and PVDF porous fibre can be obtained. With the increase of PVP, the prepared PVDF fibre diameter increases and fibre morphology is augmented.

3) Post processing of electrospun PVDF fibre: processing time is an important parameter during post processing and has great influence on forming pores. In this project, a time period of three days is the optimal choice. Fibre surface after processing is rough and pores can be observed in beads. Crystallisation in processed fibre is mainly $\beta$-phase.

\subsection{Disadvantage}

This article is based on the notion of changes in electrospinning technology effected by adjusting polymer solution concentration, solvent ratio, polymer blending and characterising PVDF porous fibre. Effects are studied and compared with the experimental results, but due to limitations of time and experiment conditions, this article cannot encompass all elements of this research area, and there are therefore some issues for further research:

This article mainly studied the effects of several important parameters (concentration, solvent ratio, solution component) on the experimental results and there are additional factors such as spinning voltage, receiving distance, surface tension of the solution and solution conductivity, temperature, environment humidity, temperature and gas atmosphere and others which have not been considered and require further study. As for the preparation of PVDF porous fibre part, properties such as mechanical properties are not compared before and after post processing. This may affect the life span of applications, which is something that can be studied at a later date.

\subsection{Future Work}

During the electrospinning process, changing the single variable will have an impact on the morphology of electrospun fibre, and the effect is not necessarily linear, so in future work, other parameters that can influence spinning should be taken into account, and combined with different parameters to find the best set for PVDF electrospinning.

Although rough surface is observed in this experiment, porous structure is not ideal. One possible reason is that in the drying process pores are collapsed and retaining porous structure during the drying process without change is problematic. Freeze-drying is an efficient method to achieve this goal, the process of which is as follows. First freeze material with water by subjecting it to below freezing point, converting water into ice, and then converting ice to steam at a higher vacuum. The material can be frozen in a freezer and then dried, or it can be frozen directly in a drying chamber and pumped quickly into a vacuum. Sublimation of water vapour is removed by a condenser and heat is usually supplied by thermal radiation. With this method, it is possible to guarantee the appearance of porous structure in the drying process, and pores will not disappear because of the collapse during the drying process. 


\section{References}

1. Al-raheil, A. and Qudah, A. M. A. (1995) 'Morphology and Melting Behaviour of Poly (butylene terephthalate )', 37, pp. 47-52.

2. Bachmann, M. et al. (1980) 'The crystal structure of phase IV of poly(vinylidene fluoride)', Journal of Applied Physics, 51(10), pp. 5095-5099. doi: 10.1063/1.327425.

3. Bachmann, M. A. and Lando, J. B. (1981) 'A Reexamination of the Crystal Structure of Phase II of Poly(vinylidene fluoride)', Macromolecules, 14(1), pp. 40-46. doi: 10.1021/ma50002a006.

4. Badalzadeh, R. et al. (2015) 'Involvement of glycogen synthase kinase-3ß and oxidation status in the loss of cardioprotection by postconditioning in chronic diabetic male rats', Advanced Pharmaceutical Bulletin, 5(3), pp. 321-327. doi: 10.15171/apb.2015.045.

5. Bogntizki, M. et al. (no date) 'Preparation of Fibers with nanoscaled morphologies - ES of Polymer Blends (PLA).pdf', 41(6).

6. Burda, C. et al. (2005) Chemistry and properties of nanocrystals of different shapes, Chemical Reviews. doi: $10.1021 / \mathrm{cr} 030063 \mathrm{a}$.

7. Chambers, S. A. (2010) 'Epitaxial growth and properties of doped transition metal and complex oxide films', Advanced Materials, 22(2), pp. 219-248. doi: 10.1002/adma.200901867.

8. Whitesides, G. M. (2005) 'Nanoscience, nanotechnology, and chemistry', Small, 1(2), pp. 172-179. doi: 10.1002/smll.200400130.

9. Guo, C., Zhou, L. and Lv, J. (2013) 'Effects of expandable graphite and modified ammonium polyphosphate on the flame-retardant and mechanical properties of wood flour-polypropylene composites', Polymers and Polymer Composites, 21(7), pp. 449-456. doi: 10.1002/app.

10. Zhao, Y. and Jiang, L. (2009) 'Hollow micro/nanomaterials with multilevel interior structures', Advanced Materials, 21(36), pp. 3621-3638. doi: 10.1002/adma.200803645.

11. Chen, Y. and Shew, C. Y. (2003) 'Conformational behavior of polar polymer models under electric fields', Chemical Physics Letters, 378(1-2), pp. 142-147. doi: 10.1016/S0009-2614(03)01208-9.

12. Sukitpaneenit, P. and Chung, T. S. (2009) 'Molecular elucidation of morphology and mechanical properties of PVDF hollow fiber membranes from aspects of phase inversion, crystallization and rheology', Journal of Membrane Science, 340(1-2), pp. 192-205. doi: 10.1016/j.memsci.2009.05.029.

13. Yee, W. A. et al. (2010) 'Supercritical carbon dioxide-treated electrospun poly(vinylidene fluoride) nanofibrous membranes: Morphology, structures and properties as an ionic-liquid host', Macromolecular Rapid Communications, 31(20), pp. 1779-1784. doi: 10.1002/marc.201000201.

14. Megelski, S. et al. (2002) 'Micro- and nanostructured surface morphology on electrospun polymer fibers', Macromolecules, 35(22), pp. 8456-8466. doi: 10.1021/ma020444a.

15. Choi, S. W. et al. (2003) 'An electrospun poly(vinylidene fluoride) nanofibrous membrane and its battery applications', Advanced Materials, 15(23), pp. 2027-2032. doi: 10.1002/adma.200304617.

16. Choi, S. W. et al. (2007) 'Characterization of electrospun PVdF fiber-based polymer electrolytes', Chemistry of Materials, 19(1), pp. 104-115. doi: 10.1021/cm060223+.

17. Kim, J. R. et al. (2004) 'Electrospun PVdF-based fibrous polymer electrolytes for lithium ion polymer batteries', Electrochimica Acta, 50(1), pp. 69-75. doi: 10.1016/j.electacta.2004.07.014.

18. Gao, K. et al. (2006) 'Crystal structures of electrospun PVDF membranes and its separator application for rechargeable lithium metal cells', Materials Science and Engineering B: Solid-State Materials for Advanced Technology, 131(1-3), pp. 100-105. doi: 10.1016/j.mseb.2006.03.035.

19. Manuscript, A. (no date) 'RSC Advances', (207890).

20. Yee, W. A. et al. (2007) 'Morphology, polymorphism behavior and molecular orientation of electrospun poly(vinylidene fluoride) fibers', Polymer, 48(2), pp. 512-521. doi: 10.1016/j.polymer.2006.11.036.

21. Greiner, A. and Wendorff, J. H. (2007) 'Electrospinning: A fascinating method for the preparation of ultrathin fibers', Angewandte Chemie - International Edition, 46(30), pp. 5670-5703. doi: 10.1002/anie.200604646.

22. CM, L. (2003) 'Nanoscale science and technology: building a big future from small things', MRS Bull., 28(July 2003), p. 486. 
23. Crecorio, R. and Cestari, M. (1994) 'Eff e d of Crystallization Temperature on the Crystalline Phase Content and Morphology of Poly ( vinylidene Fluoride )', Journal of Polymer Science: Part B: Polymer Physics, Vol. 32, pp. 859-870. doi: 10.1002/polb.1994.090320509.

24. Ejaz Ahmed, F. et al. (2014) 'Underwater superoleophobic cellulose/electrospun PVDF-HFP membranes for efficient oil/water separation', Desalination. Elsevier B.V., 344, pp. 48-54. doi: 10.1016/j.desal.2014.03.010.

25. Esterly, D. M. and Love, B. J. (2004) 'Phase Transformation to Beta-Poly ( vinylidene fluoride ) by Milling', Journal of Polymer Science Part B: Polymer Physics, 42(1), pp. 91-97.

26. Fong, H. and Reneker, D. H. (1999) 'Elastomeric Nanofibers of SBS Triblock Copolymer Elastomeric Nanofibers of Styrene - Butadiene - Styrene', Journal of Polymer Science Part B: Polymer Physics, (January 1999), p. 3488.

27. For, C. and Flyback, F. (1972) 'Elite Sttes ate'.

28. Geoffrey Taylor (no date) 'Desintegration of water drops in an electric field'.

29. Gregorio, R. (2006) 'Determination of the ??,??, and ?? crystalline phases of poly(vinylidene fluoride) films prepared at different conditions', Journal of Applied Polymer Science, 100(4), pp. 3272-3279. doi: 10.1002/app.23137. 